KANAZAWA-02-14

July, 2002

\title{
$\mu$-term as the origin of baryon and lepton number asymmetry
}

\author{
Daijiro Suematsu * \\ Institute for Theoretical Physics, Kanazawa University, \\ Kanazawa 920-1192, JAPAN
}

\begin{abstract}
We study a possibility of combining an origin of the $\mu$-term and the baryon and lepton number asymmetry. If we assume that the $\mu$-term is generated through a flat direction of a singlet scalar field, the coherent oscillation of this condensate around its potential minimum can store the global U(1) charge asymmetry. The decay of this condensate can distribute this asymmetry into the lepton and baryon number asymmetry as far as its decay occurs at an appropriate temperature. We examine the compatibility between this scenario and the small neutrino mass generation based on both the ordinary seesaw mechanism and the bilinear $R$-parity violating terms.
\end{abstract}

*e-mail:suematsu@hep.s.kanazawa-u.ac.jp 


\section{Introduction}

In the present astroparticle physics, it is one of the crucial problems to clarify an origin of the baryon number $(B)$ asymmetry in the universe [1]. It has been suggested that the electroweak sphaleron in the standard model (SM) plays an important role in that investigation [2]. Since the $B$ asymmetry generated at high energy scales can be washed out by this effect unless $B-L \neq 0$, we need a suitable scenario to escape this problem.

Leptogenesis seems to present an elegant scenario for the explanation of the $B$ asymmetry on the basis of the lepton number $(L)$ violation [3]. Since the electroweak sphaleron interaction conserves $B-L$, a part of the $L$ asymmetry can be converted into the $B$ asymmetry through this interaction. Recent experiments related to the neutrino oscillation suggest the existence of small neutrino masses $[4,5]$. This fact seems to indicate the existence of the $B-L$ violation at a certain high energy scale as far as we consider to explain the small neutrino masses by the seesaw mechanism. From this point of view the leptogenesis scenario is very promising as an origin of the $B$ asymmetry.

Several leptogenesis scenarios have been proposed under the various $L$ violating schemes $[3,6]$. In the supersymmetric model, one may consider the $L$ violation due to some condensates along a flat direction. As such scenarios there are, for example, the decay of the Affleck-Dine (AD) condensate [7] in the $\mathrm{LH}_{2}$ direction [8] and also the decay of the right-handed sneutrino condensate in the chaotic inflation scenario $[9,10]$.

As an extension of this kind of leptogenesis scenario, we may consider a possibility that an additional global $\mathrm{U}(1)_{X}$ charge asymmetry is generated due to the AD mechanism and then it is converted into the $B$ and $L$ asymmetry through the sphaleron interaction. This can occur if an interaction which violates both $\mathrm{U}(1)_{X}$ and $B-L$ symmetry is in the thermal equilibrium. ${ }^{1}$ In this paper, we would like to propose such a model which can also be related to the origin of the $\mu$-term $\mu H_{1} H_{2}$.

In the minimal supersymmetric standard model (MSSM) the origin of a scale of the $\mu$-term is not known. Since it is a supersymmetric mass term, we have no reason why it takes a similar value to the supersymmetry (SUSY) breaking scale. Fixing its scale to the weak scale is called the $\mu$-problem $[14,15]$. Usually the $\mu$-term is considered to be spontaneously generated through, for example, a vacuum expectation value (VEV)

\footnotetext{
${ }^{1}$ Similar scenario is proposed in some works in the different context $[11,12,13]$.
} 
of some SM singlet scalar field $S$ as $\mu=\lambda\langle S\rangle .^{2}$ If this singlet field has an almost flat direction and stores a large energy due to the deviation from its true vacuum value at a suitable period of the expansion of the universe, ${ }^{3}$ this condensate may store the sufficient $\mathrm{U}(1)_{X}$ charge asymmetry during the oscillation due to the AD mechanism. The decay of this condensate through the $\mathrm{U}(1)_{X}$ invariant coupling $\lambda S H_{1} H_{2}$ produces the $\mathrm{U}(1)_{X}$ charge asymmetry in the Higgsino sector $\tilde{H}_{1,2}$ and it is distributed into other fields in the thermal equilibrium. If both the electroweak sphaleron interaction and a certain interaction violating simultaneously both $\mathrm{U}(1)_{X}$ and $B-L$ are in the thermal equilibrium at this period, we expect that a part of this asymmetry is converted into the $B$ and $L$ asymmetry.

This scenario is largely affected by both temperatures at which the $B-L$ violating interaction and the soft SUSY breaking effects leave the thermal equilibrium. Additionally, the true VEV of $S$ and its coupling $\lambda$ to the Higgsinos are constrained by the scale of $\mu$. We need to study a viability of this scenario under these constraints. Moreover, the small mass generation of the neutrinos has an intimate connection to this scenario as the origin of the $B-L$ violation, although the situation seems to be completely dependent on the model. For example, one may consider scenarios based on the usual seesaw mechanism or the bilinear $R$-parity violating terms $\epsilon_{\alpha} L_{\alpha} H_{2}$. In the former case, it is well known that the lepton number violating effective interaction appears incidentally at a certain high energy scale. It can leave the thermal equilibrium at a rather high temperature. In the latter case, various works suggest that the bilinear $R$-parity violating terms can be successfully related to the neutrino masses $[18,19,20]$. However, if the Higgsino-neutrino mixing induced by the bilinear $R$-parity violating terms is in the thermal equilibrium around the weak scale, the produced $L$ and $B$ asymmetry may be completely washed out. Thus, it is worthy to study what kind of neutrino mass generation mechanisms are compatible with this scenario.

This paper is organized as follows. In section 2 we discuss the production of the global $\mathrm{U}(1)_{X}$ charge asymmetry. In section 3 its conversion into the $L$ and $B$ asymmetry is studied. In section 4 we discuss the relation of this scenario to the neutrino mass. Section

\footnotetext{
${ }^{2}$ In such a case, it is well-known that there can be also the Peccei-Quinn symmetry as an additional global symmetry.

${ }^{3}$ Inflation models based on this behavior of $S$ have been considered in $[16,17]$. In this paper the inflation is assumed to be induced by other scalar field.
} 
5 is devoted to the summary.

\section{Production of the $\mathrm{U}(1)_{X}$ charge asymmetry}

To present the basic idea of the scenario, we consider a model defined by the superpotential $W_{\text {MSSM }}+W_{1}$. $W_{\text {MSSM }}$ is the superpotential for the MSSM Yukawa interactions such as

$$
W_{\mathrm{MSSM}}=y_{U}^{\alpha \beta} Q_{\alpha} \bar{U}_{\beta} H_{2}+y_{D}^{\alpha \beta} Q_{\alpha} \bar{D}_{\beta} H_{1}+y_{E}^{\alpha \beta} L_{\alpha} \bar{E}_{\beta} H_{1}
$$

For the construction of an additional superpotential $W_{1}$ which plays the essential role in the scenario, we introduce the massless SM singlet chiral superfield $S$. As such a superpotential we suppose

$$
W_{1}=\lambda S H_{1} H_{2}+\frac{d}{M_{\mathrm{pl}}^{N-3}} S^{N},
$$

where $\lambda$ and $d$ is assumed to be real and $d=O(1)$. This superpotential can be realized by imposing a suitable symmetry. We discuss this point in appendix A.

In this model we find that there are two new abelian global symmetries $\mathrm{U}(1)_{\mathrm{PQ}}$ and $\mathrm{U}(1)_{R}$ other than the $B$ and $L$ symmetries if we neglect the nonrenormalizable term in $W_{1}$. They are defined in Table 1. Among these four Abelian symmetries there remain two global symmetries $\mathrm{U}(1)_{B-L}$ and $\mathrm{U}(1)_{X}$ as those with no $\mathrm{SU}(3)$ and $\mathrm{SU}(2)$ gauge anomaly. It is easily checked that the $\mathrm{U}(1)_{X}$ charge $X$ can be represented as the linear combination of the four global Abelian charges as $X=B+L-10 Q_{\mathrm{PQ}}+3 Q_{R}$.

Now we consider the generation of the $\mathrm{U}(1)_{X}$ charge asymmetry. For this study we need to note that $S$ gives a $D$-flat direction. The VEV is generally supposed to be complex. The direction described by $\langle S\rangle$ is slightly lifted by the nonrenormalizable term in the scalar potential induced by the last term of $W_{1}$. In the early universe there are additional effective contributions to the scalar potential induced by the SUSY breaking effects caused by the large Hubble constant $H$ [21] and the thermal effects [22] other than the ordinary soft SUSY breaking. If we take account of these effects and we put $\langle S\rangle \equiv u e^{i \theta}$, the scalar potential is found to be expressed as

$$
\begin{aligned}
V \simeq\left(-c H^{2}\right. & \left.+M_{S}^{2}(T)\right) u^{2}+\frac{|d|^{2} N^{2}}{M_{\mathrm{pl}}^{2 N-6}} u^{2 N-2} \\
& +\left\{\left(\frac{a m_{3 / 2} e^{i \theta_{a}}}{M_{\mathrm{pl}}^{N-3}}+\frac{b H e^{i \theta_{b}}}{M_{\mathrm{pl}}^{N-3}}\right) u^{N} e^{i N \theta}+\text { h.c. }\right\}
\end{aligned}
$$




\begin{tabular}{c|ccccccccccc} 
& $\tilde{g}, \tilde{W}, \tilde{B}$ & $Q_{L}$ & $\bar{U}_{L}$ & $\bar{D}_{L}$ & $L_{L}$ & $\bar{E}_{L}$ & $H_{1}$ & $H_{2}$ & $S$ & $A_{S U(3)}$ & $A_{S U(2)}$ \\
\hline$Q_{\mathrm{PQ}}$ & 0 & 0 & -2 & 1 & -1 & 2 & -1 & 2 & -1 & $-\frac{3}{2}$ & -1 \\
$Q_{R}$ & -1 & $-\frac{1}{6}$ & -1 & 0 & $-\frac{5}{6}$ & $\frac{2}{3}$ & $-\frac{5}{6}$ & $\frac{1}{6}$ & $-\frac{1}{3}$ & -5 & $-\frac{13}{3}$ \\
$B$ & 0 & $\frac{1}{3}$ & $-\frac{1}{3}$ & $-\frac{1}{3}$ & 0 & 0 & 0 & 0 & 0 & 0 & $\frac{3}{2}$ \\
$L$ & 0 & 0 & 0 & 0 & 1 & -1 & 0 & 0 & 0 & 0 & $\frac{3}{2}$ \\
\hline
\end{tabular}

Table 1 Global U(1) charge assignment and gauge anomaly.

where $m_{3 / 2}$ is a typical soft SUSY breaking scale of $O(1) \mathrm{TeV}$ and the coefficients $a, b$ and $c$ are $O(1)$ real constants. The $\mathrm{CP}$ phases $\theta_{a}$ and $\theta_{b}$ in the curly brackets are induced by the above mentioned SUSY breaking effects which violate the $\mathrm{U}(1)_{X}$. The effective mass $M_{S}^{2}(T)$ contains the usual soft SUSY breaking mass $m_{S}^{2}$ of $O\left(m_{3 / 2}^{2}\right)$ and the thermal mass $\sim \lambda^{2} T^{2}$ caused by the coupling of $S$ with $H_{1,2}$ in the thermal plasma. It can be expressed depending on the value of $u$ as [22]

$$
M_{S}^{2}(T) \simeq \begin{cases}m_{S}^{2} & (\lambda u>T), \\ m_{S}^{2}+\lambda^{2} T^{2} & (\lambda u<T) .\end{cases}
$$

The Hubble constant contribution $H^{2}$ dominates the mass of the condensate during the inflation. Thus, if the sign of the Hubble constant contribution in eq. (3) is negative $(c>0)[21]$, the magnitude of the condensate takes a large value such as

$$
u_{I} \simeq\left(H M_{\mathrm{pl}}^{N-3}\right)^{\frac{1}{N-2}}
$$

On the other hand, the phase $\theta$ of the condensate at the potential minimum takes one of the $N$ distinct values $\theta=-\theta_{b} / N+2 \pi \ell / N(\ell=1,2, \cdots, N)$. At this period the condensate follows this instantaneous potential minimum since its evolution is almost the critical damping. The dilute plasma appears as a result of a partial decay of the inflaton. Then the temperature rapidly increases to $T_{\max } \simeq\left(T_{R}^{2} H M_{\mathrm{pl}}\right)^{1 / 4}[22] . T_{R}$ is the reheating temperature realized after the completion of the inflaton decay and can be expressed as $T_{R} \simeq \sqrt{M_{\mathrm{pl}} \Gamma_{I}}$ where $\Gamma_{I}$ is the inflaton decay width. If this temperature $T_{\max }$ does not satisfy $\lambda\left|u_{I}\right|<T_{\max }$, no thermal contribution to $M_{S}^{2}(T)$ appears and $M_{S}^{2}(T)$ takes the expression of the upper one in eq. (4) [21, 22]. Thus, the condition for the thermal effects to be negligible during the inflation gives the following lower bound on $\lambda$ :

$$
\lambda>T_{R}^{1 / 2} H_{I}^{\frac{N-6}{4(N-2)}} M_{\mathrm{pl}}^{\frac{10-3 N}{4(N-2)}},
$$


where $H_{I}$ is the Hubble parameter during the inflation.

When the inflaton evolves and $H$ decreases to $H \sim m_{3 / 2}$, the effective squared mass of the condensate becomes positive and then $u=0$ is the minimum of the scalar potential $V$. The condensate starts to oscillate around $u=0$, and the thermal effects due to the dilute plasma to $M_{S}^{2}(T)$ appears. Then $M_{S}^{2}(T)$ takes the form of the lower one in eq. (4). At this time, the dominant term for the $\mathrm{U}(1)_{X}$ breaking changes from the second term in the curly brackets of eq. (3) to the first term. Since the phase $\theta_{a}$ and $\theta_{b}$ are generally independent, the phase $\theta$ of the condensate changes non-adiabatically from that determined by $\theta_{b}$ to that determined by $\theta_{a}$ due to the torque in the angular direction. Thus, the $X$ asymmetry is stored in the condensate during its evolution due to the AD mechanism [7].

This $X$ asymmetry can be estimated by taking account that the $X$ current conservation is violated by the dominant $X$ breaking term in the curly brackets in eq. (3) as

$$
\frac{d \delta n_{X}(t)}{d t}=X(S) \frac{a m_{3 / 2} u^{N}}{M_{\mathrm{pl}}^{N-3}} \sin \delta,
$$

where $X(S)$ is the $X$ charge of $S$ and $\delta$ is determined by the difference of $\theta_{a}$ and $\theta_{b}$. By solving this equation, the $X$ asymmetry produced at this period is found to be roughly expressed as $[7,21,22]^{4}$

$$
\delta n_{X}(t) \simeq X(S) \frac{m_{3 / 2}}{H} H^{\frac{N}{N-2}} M_{\mathrm{pl}}^{\frac{2(N-3)}{N-2}} \sin \delta
$$

where $t$ is the time when $H \sim m_{3 / 2}$. Following this, the reheating due to the inflaton decay is completed at $H \sim \Gamma_{I}{ }^{5}$ On the other hand, the stored $X$ asymmetry in the condensate is liberated into the thermal plasma through the decay of the condensate by the $X$ conserving coupling $\lambda S H_{1} H_{2}$ in $W_{1}$. Since the oscillation behaves as a matter for the expansion of the universe, it can dominate the energy density of the universe before its decay which occurs at $H \sim \Gamma_{S}$. For the reasonable value of $\lambda$, this is the case since $\Gamma_{S}<\Gamma_{I}$ is satisfied. Taking account of this, we estimate the ratio of the $X$ asymmetry to the entropy density $s$ at this period as

$$
\frac{\delta n_{X}\left(\tilde{t}_{R}\right)}{s} \simeq \frac{\delta n_{X}(t)}{\tilde{T}_{R}^{3}} \frac{t^{2}}{\tilde{t}_{R}^{2}} \simeq X(S) \tilde{T}_{R} m_{3 / 2}^{\frac{4-N}{N-2}} M_{\mathrm{pl}}^{\frac{-2}{N-2}} \sin \delta,
$$

\footnotetext{
${ }^{4}$ The rigorous estimation requires the numerical calculation as discussed in [21]. It is beyond the scope of this paper and we do not go further here.

${ }^{5}$ Here we assume that $m_{3 / 2}>\Gamma_{I}$ is satisfied. This means $T_{R}<\sqrt{M_{\mathrm{pl}} m_{3 / 2}} \simeq 10^{11} \mathrm{GeV}$.
} 
where we use $\tilde{t}_{R} \sim \Gamma_{S}^{-1} \sim M_{\mathrm{pl}} / \tilde{T}_{R}^{2}$. If the temperature $\tilde{T}_{R}\left(\simeq 10^{10} \lambda \mathrm{GeV}\right)$ is appropriate to keep the $X$ asymmetry and convert it into the $B$ asymmetry through the sphaleron interaction, we can obtain the $B$ asymmetry. ${ }^{6}$

Finally we discuss the evolution of $u$ after this period to see the relation of this scenario to the $\mu$-term. We assume $H_{I} \sim 10^{13} \mathrm{GeV}$ during the inflation on the basis of the CMB data and $T_{R} \lesssim 10^{9} \mathrm{GeV}$. For these values we obtain $T_{\max } \sim 10^{13} \mathrm{GeV}$. Thus, if we take $N=$ 4 in $W_{1}$, as an example, eq. (5) gives $u_{I} \sim 10^{16} \mathrm{GeV}$ and eq. (6) suggests that $\lambda \gtrsim 10^{-3.5}$ should be satisfied. When the temperature decreases from $T_{\max }$ to $T_{c} \sim m_{3 / 2} / \lambda, M_{S}^{2}(T)$ represented by the lower one in eq. (4) starts to be dominated by the soft supersymmetry breaking mass $m_{S}^{2}$. If $m_{S}^{2}<0$ is realized by some reason [24], $u \neq 0$ becomes the true vacuum after this period. Since the $\mu$-term is generated from the first term in $W_{1}$ as $\mu=\lambda u$, such a value of $u$ should be $u_{0} \lesssim 10^{6} \mathrm{GeV}$ to realize the appropriate $\mu$ for the above mentioned $\lambda .{ }^{7}$ Although the condensate again starts to oscillate around $u_{0}$, the deviation from $u_{0}$ instantaneously decays into the light fields through the $X$ conserving coupling $S H_{1} H_{2}$ since $H<\Gamma_{S}$ is satisfied at this time. The released energy cannot dominate the total energy density $\left(\frac{\pi^{2}}{30} g_{*} T^{4} \gg m_{3 / 2}^{2} u_{0}^{2}\right)$ and then the effects of the produced entropy is negligible. The $X$ asymmetry obtained in eq. (9) can be used as the origin of the $B$ asymmetry.

\section{Generation of $B$ asymmetry}

Next we examine whether this $X$ asymmetry transformed into the thermal plasma through the decay of the condensate can remain as a nonzero value and be partially converted into the $B$ asymmetry. This should be studied taking account that the electroweak sphaleron interaction and other various interactions are in the thermal equilibrium. For this study, it is convenient to consider the detailed balance of these interactions and solve the chemical equilibrium equations [26, 27]. The particle-antiparticle number asymmetry $\delta n_{f}$ can be approximately related to the corresponding chemical potential $\mu_{f}$. In the case of $\mu_{f} \ll T$,

\footnotetext{
${ }^{6}$ This $\tilde{T}_{R}$ is the marginal value for the cosmological gravitino problem [23].

${ }^{7}$ Such a $u_{0}$ may be expected to be determined either by the nonrenormalizable terms or by the pure radiative symmetry breaking effect in which $u_{0}$ is estimated by using the renormalization group equation as discussed in [25].
} 
it can be represented as

$$
\delta n_{f} \equiv n_{f}-n_{f^{c}}= \begin{cases}\frac{g_{f}}{6} T^{2} \mu_{f} & (f: \text { fermion }), \\ \frac{g_{f}}{3} T^{2} \mu_{f} & (f: \text { boson }),\end{cases}
$$

where $g_{f}$ is a number of relevant internal degrees of freedom of the field $f$. By solving the detailed valance equations for the chemical potential $\mu_{f}$, we can study the charge asymmetry at the period after the decay of the condensate.

If the $\mathrm{SU}(2)$ and $\mathrm{SU}(3)$ sphaleron interactions are in the thermal equilibrium, we have the conditions such as

$$
\begin{aligned}
& \sum_{i=1}^{N_{g}}\left(3 \mu_{Q_{i}}+\mu_{L_{i}}\right)+\mu_{\tilde{H}_{1}}+\mu_{\tilde{H}_{2}}+4 \mu_{\tilde{W}}=0, \\
& \sum_{i=1}^{N_{g}}\left(2 \mu_{Q_{i}}-\mu_{U_{i}}-\mu_{D_{i}}\right)+6 \mu_{\tilde{g}}=0,
\end{aligned}
$$

where $N_{g}$ is a number of the generation of quarks and leptons. The cancellation of the total hypercharge or the electric charge of plasma in the universe requires

$$
\begin{aligned}
\sum_{i=1}^{N_{g}}\left(\mu_{Q_{i}}+\right. & \left.2 \mu_{U_{i}}-\mu_{D_{i}}-\mu_{L_{i}}-\mu_{E_{i}}\right)+\mu_{\tilde{H}_{2}}-\mu_{\tilde{H}_{1}} \\
& +2 \sum_{i=1}^{N}\left(\mu_{\tilde{Q}_{i}}+2 \mu_{\tilde{U}_{i}}-\mu_{\tilde{D}_{i}}-\mu_{\tilde{L}_{i}}-\mu_{\tilde{E}_{i}}\right)+2\left(\mu_{H_{2}}-\mu_{H_{1}}\right)=0 .
\end{aligned}
$$

When Yukawa interactions in $W_{\mathrm{MSSM}}+W_{1}$ are in the thermal equilibrium, they impose the conditions ${ }^{8}$

$$
\begin{array}{ll}
\mu_{Q_{i}}-\mu_{U_{j}}+\mu_{H_{2}}=0, & \mu_{Q_{i}}-\mu_{D_{j}}+\mu_{H_{1}}=0, \\
\mu_{L_{i}}-\mu_{E_{j}}+\mu_{H_{1}}=0, & \mu_{S}+\mu_{\tilde{H}_{1}}+\mu_{\tilde{H}_{2}}=0 .
\end{array}
$$

There are also the conditions for the gauge interactions in the thermal equilibrium, which are summarized as

$$
\mu_{\tilde{Q}_{i}}=\mu_{\tilde{g}}+\mu_{Q_{i}}=\mu_{\tilde{W}}+\mu_{Q_{i}}=\mu_{\tilde{B}}+\mu_{Q_{i}} .
$$

The similar relations to eq. (15) is satisfied for leptons $L_{i}$, Higgs $H_{1,2}$ and other fields $U_{i}, D_{i}, E_{i}$ which have the SM gauge interactions. Flavor mixings of quarks and leptons

\footnotetext{
${ }^{8}$ We should note that the second term in $W_{1}$ leaves the thermal equilibrium at $T \sim M_{\mathrm{pl}}$. Since $S$ has no other coupling to the MSSM contents than $\lambda S H_{1} H_{2}$, the last one in eq. (14) is the only condition for $\mu_{S}$.
} 
due to the Yukawa couplings allow us to consider the flavor independent chemical potential such as $\mu_{Q}=\mu_{Q_{i}}$ and $\mu_{L}=\mu_{L_{i}}$.

Here we introduce a term violating both $B-L$ and $X$, which is necessary to convert the $X$ asymmetry into the $B$ and $L$ asymmetry. If such a term exists, only a linear combination of these two $\mathrm{U}(1) \mathrm{s}$ is absolutely conserved. Then a part of $X$ asymmetry can be converted into the $B-L$ asymmetry. As an interesting example, we may consider a term $\left(\mathrm{LH}_{2}\right)^{k}$. It corresponds to the effective neutrino mass term of the ordinary seesaw mechanism in the $k=2$ case and also the bilinear $R$-parity violating term in the $k=1$ case. The thermal equilibrium condition of these terms can be written as

$$
\mu_{L}+\mu_{H_{2}}=0 \quad(k=2), \quad \mu_{L}+\mu_{\tilde{H}_{2}}=0 \quad(k=1) .
$$

We find that there is an independent chemical potential in these thermal equilibrium conditions $(11) \sim(16)$. It can be taken as $\mu_{\tilde{H}_{2}}$, which corresponds to the above mentioned remaining symmetry.

By now we have not taken account of the equilibrium conditions for the soft SUSY breaking terms. The soft SUSY breaking terms are in the thermal equilibrium when $H \lesssim \Gamma_{s s}$ is satisfied. Here the rate of the soft SUSY breaking effects is written as $\Gamma_{s s} \simeq$ $m_{3 / 2}^{2} / T[12]$. From this we find that the soft SUSY breaking effects are in the thermal equilibrium for the temperature $T \lesssim T_{s s} \simeq 10^{7} \mathrm{GeV}$. Thus, for $T \lesssim T_{s s}$ we find that $\mu_{\tilde{g}}=0$ is satisfied and then eqs. $(11) \sim(16)$ result in $\mu_{\tilde{H}_{2}}=0$. The $X$ asymmetry produced through the decay of the condensate disappears in this case. In order to escape this, if we define $T_{X}$ as a temperature at which the $X$ and $B-L$ violating interaction is out-of-equilibrium, we need to require that $T_{X}$ should satisfy $T_{s s} \lesssim T_{X} \lesssim \tilde{T}_{R}$. We will discuss this condition in the next section. If these conditions are satisfied, the $X$ asymmetry induced through the decay of the condensate can be partially converted into the $B$ asymmetry.

By solving eqs. $(11) \sim(16), \mu_{Q}, \mu_{L}, \mu_{H_{1,2}}$ and $\mu_{\tilde{g}}$ can be written with the chemical potential of Higgsino field $\tilde{H}_{2}$ at $T_{X}$ in such a way as

$$
\begin{aligned}
& \mu_{Q}=\frac{17 N_{g}+6}{N_{g}\left(10 N_{g}^{2}-17 N_{g}-15\right)} \mu_{\tilde{H}_{2}}, \quad \mu_{L}=-\mu_{H_{2}}=\frac{5\left(4 N_{g}+3\right)}{10 N_{g}^{2}-17 N_{g}-15} \mu_{\tilde{H}_{2}}, \\
& \mu_{H_{1}}=-\frac{40 N_{g}+3}{10 N_{g}^{2}-17 N_{g}-15} \mu_{\tilde{H}_{2}}, \quad \mu_{\tilde{g}}=-\frac{\left(10 N_{g}+3\right) N_{g}}{10 N_{g}^{2}-17 N_{g}-15} \mu_{\tilde{H}_{2}},
\end{aligned}
$$


for the $k=2$ case and

$$
\begin{aligned}
& \mu_{Q}=\frac{2 N_{g}^{3}+N_{g}^{2}+17 N_{g}+6}{N_{g}\left(6 N_{g}^{2}-17 N_{g}-15\right)} \mu_{\tilde{H}_{2}}, \quad \mu_{L}=-\mu_{\tilde{H}_{2}}, \quad \mu_{H_{2}}=-\frac{4 N_{g}^{2}+20 N_{g}+15}{6 N_{g}^{2}-17 N_{g}-15} \mu_{\tilde{H}_{2}}, \\
& \mu_{H_{1}}=\frac{4 N_{g}^{2}-40 N_{g}-3}{6 N_{g}^{2}-17 N_{g}-15} \mu_{\tilde{H}_{2}}, \quad \mu_{\tilde{g}}=-\frac{\left(10 N_{g}+3\right) N_{g}}{6 N_{g}^{2}-17 N_{g}-15} \mu_{\tilde{H}_{2}},
\end{aligned}
$$

for the $k=1$ case. Defining $B$ and $L$ as $\delta n_{B} \equiv B T^{2} / 6$ and $\delta n_{L} \equiv L T^{2} / 6$, we can calculate these values at $T_{s s}$ by using eq. (10), (17) and (18) as

$$
\begin{aligned}
& k=2:\left\{\begin{array}{l}
B=\frac{80 N_{g}^{3}+204 N_{g}^{2}-150 N_{g}-72}{360 N_{g}^{3}+3308 N_{g}^{2}-1419 N_{g}-1143} X, \\
L=\frac{N_{g}\left(60 N_{g}^{2}-42 N_{g}-126\right)}{360 N_{g}^{3}+3308 N_{g}^{2}-1419 N_{g}-1143} X,
\end{array}\right. \\
& k=1:\left\{\begin{array}{l}
B=\frac{56 N_{g}^{3}+192 N_{g}^{2}-150 N_{g}-72}{378 N_{g}^{3}+3822 N_{g}^{2}-624 N_{g}-1143} X, \\
L=\frac{N_{g}\left(92 N_{g}^{2}-15 N_{g}-126\right)}{378 N_{g}^{3}+3822 N_{g}^{2}-624 N_{g}-1143} X,
\end{array}\right.
\end{aligned}
$$

where $X$ is defined as $\delta n_{X} \equiv X T^{2} / 6$. These results show that all of $B, L$ and $B-L$ take nonzero values as far as $X \neq 0$.

When the temperature goes below $T_{s s}$, the soft SUSY breaking terms are in the thermal equilibrium. This results in $\mu_{\tilde{g}}=0$ and $X=0$. However, if the $X$ and $B-L$ violating interaction is assumed to be out-of-equilibrium at $T_{s s}$, the equilibrium conditions are represented by $(11) \sim(15)$. Thus the $B-L$ asymmetry existing at $T_{s s}$ is kept after this period. The equilibrium conditions give the ordinary MSSM values for $B$ and $L$ as

$$
B=\frac{4\left(2 N_{g}+1\right)}{22 N_{g}+13}(B-L), \quad L=-\frac{14 N_{g}+9}{22 N_{g}+13}(B-L),
$$

where we should use the $B-L$ value obtained from eq. (19) or (20). The $B$ asymmetry produced in this scenario is finally estimated as

$$
Y_{B} \equiv \frac{\delta n_{B}}{s}=\frac{\delta n_{X}}{s} \frac{B-L}{X} \frac{4\left(2 N_{g}+1\right)}{22 N_{g}+13} \kappa \simeq \tilde{T}_{R} m_{3 / 2}^{\frac{4-N}{N-2}} M_{\mathrm{pl}}^{\frac{-2}{N-2}} f\left(N_{g}\right) \kappa \sin \delta,
$$

where $\kappa(\leq 1)$ is introduced to take account of the washout effect which we will discuss later. $f\left(N_{g}\right)$ is a numerical factor taking $f(3) \simeq 0.3$ and 0.05 for $k=2$ and $k=1$, respectively. From this result, we find that this scenario can produce the presently observed $B$ asymmetry $Y_{B}=(0.6-1) \times 10^{-10}$ as far as $N \geq 5$ for $\tilde{T}_{R}>10^{7} \mathrm{GeV}$. Since the $X$ asymmetry disappears at the temperature less than $T_{s s}$ through the effects of the soft SUSY breakings, the $X$ asymmetry cannot be related to the dark matter abundance in the universe [11]. 
(a) \begin{tabular}{c|cc} 
& $Q_{\mathrm{PQ}}$ & $Q_{R}$ \\
\hline$\overline{\mathcal{N}}$ & 0 & $-\frac{1}{2}$
\end{tabular}

\begin{tabular}{c|cc} 
& $Q_{\mathrm{PQ}}$ & $Q_{R}$ \\
\hline$\overline{\mathcal{N}}$ & $-\frac{1}{3}$ & $-\frac{3}{4}$ \\
$\mathcal{N}$ & $\frac{1}{2}$ & $\frac{1}{4}$
\end{tabular}

Table 2 The $\mathrm{U}(1)$ charge assignments for $\overline{\mathcal{N}}$ and $\mathcal{N}$, whose $B$ and $L$ are zero.

\section{Relation to neutrino masses}

It is an interesting and important issue to consider the compatibility of this scenario for the generation of the $B$ and $L$ asymmetry with the small neutrino mass generation. This is because the $X$ and $B-L$ violation is considered to be introduced through the interaction related to the neutrino masses as mentioned before. In this section we study two typical schemes for the neutrino mass generation in this scenario. For this purpose we introduce SM singlet chiral superfields $\overline{\mathcal{N}}$ and $\mathcal{N}$ which have the global U(1) charges listed in Table. 2.

\subsection{Ordinary see-saw scenario}

First we consider the lepton sector characterized by the renormalizable superpotential

$$
W_{2}=y_{N}^{\alpha} L_{\alpha} \overline{\mathcal{N}} H_{2}+M_{R} \overline{\mathcal{N}}^{2}+\cdots
$$

Here we assume the (a) type charge assignment in Table 2 and abbreviate the irrelevant terms. ${ }^{9}$ The first term violates both $\mathrm{U}(1)_{B-L}$ and $\mathrm{U}(1)_{X}$. If we integrate out the righthanded neutrino $\overline{\mathcal{N}}$ following the usual seesaw scheme, we have the effective $X$ violating interaction which corresponds to the $k=2$ case discussed in the previous section. The necessary condition for the applicability of this scenario is that this effective interaction should have left the thermal equilibrium by $T_{s s}$. By using the right-handed neutrino mass $M_{R}$, we can summarize this condition such that $H>T_{X}^{3} / M_{R}^{2}$ should be satisfied at $T_{X} \gtrsim T_{s s}$. This results in $M_{R} \gtrsim 10^{12} \mathrm{GeV}$ which is a suitable value for the explanation of the neutrino masses required by the neutrino oscillation data $[4,5]$.

\footnotetext{
${ }^{9}$ There is another possible term $\overline{\mathcal{N}}^{3}$, which violates $\mathrm{U}(1)_{R}$ and is the same order as the presented one. However, it brings no influence for the present argument.
} 
In this type of seesaw model the leptogenesis is usually considered on the basis of the out-of-equilibrium decay of the heavy right-handed neutrinos or the decay of sneutrino condensate. However, if we consider the spontaneous $\mu$-term generation along the almost flat direction of $\langle S\rangle$ as discussed here, the $B$ asymmetry produced by this usual leptogenesis might not be the dominant one. As mentioned before, we assume that the decay of the condensate is completed above the temperature $T_{X}$ which can be sufficiently lower than the masses of the right-handed heavy neutrinos. Then the $B$ asymmetry produced through the usual scenario seems to be washed out or overridden by the $B$ asymmetry produced in the present scenario. If there is an additional entropy production after the thermal decoupling of the weak sphaleron interaction, such a reheating temperature $T_{N}$ should satisfy $T_{N} \gtrsim 1 \mathrm{MeV}$ because of the nucleosynthesis requirement. Since the dilution effect $\kappa$ due to this entropy release is written as $\kappa \sim T_{N} / T_{W}$ where $T_{W} \simeq 100 \mathrm{GeV}$, we can estimate it as $\kappa \gtrsim 10^{-5}$. Thus the baryon number asymmetry produced by the present scenario with an appropriate value of $N$ can be sufficient for the explanation of the baryon number in the universe. This kind of possibility may be promising as much as the usual leptogenesis scenario.

\subsection{Bilinear $R$-parity violating scenario}

Another scheme for the small neutrino mass may be characterized by the bilinear $R$-parity violating term in the superpotential such as

$$
W_{3}=\epsilon_{\alpha} L_{\alpha} H_{2}
$$

which violates both $\mathrm{U}(1)_{B-L}$ and $\mathrm{U}(1)_{X}$. In the MSSM with $W_{3}$ we can show that the small neutrino mass generation is possible due to the neutralino-neutrino mixing. We discuss this point in the appendix B.

If bilinear $R$-parity violating term is simultaneously in the thermal equilibrium with the soft SUSY breaking terms, the chemical detailed balance equations result in $\mu_{\tilde{H}_{2}}=0$. In order to keep nonzero $B-L$ asymmetry, the $\epsilon_{\alpha} L_{\alpha} H_{2}$ term should be in the thermal equilibrium at $T \gtrsim T_{s s}$ but be out-of-equilibrium at $T<T_{s s}$. We can show that such a required behavior of the bilinear $R$-parity violating term is realizable by presenting a simple example. We assume the type (b) charge assignment for the SM singlet chiral superfields $\mathcal{N}$ and $\overline{\mathcal{N}}$. If we impose either $\mathrm{U}(1)_{\mathrm{PQ}}$ or $\mathrm{U}(1)_{R}$ invariance for the MSSM 
contents, we have the superpotential

$$
W_{4}=\hat{c}_{1} \frac{\overline{\mathcal{N}}^{3}}{M^{2}} L H_{2}+\hat{c}_{2} \frac{(\overline{\mathcal{N}} \mathcal{N})^{2}}{M}++\hat{c}_{3} \frac{\overline{\mathcal{N}}^{3} \mathcal{N}^{2}}{M^{2}}+\cdots,
$$

where $\hat{c}_{1,2,3}$ are $O(1)$ constants and the ellipses represent higher order terms. The first term violates both $\mathrm{U}(1)_{B-L}$ and $\mathrm{U}(1)_{X}$. If we introduce the soft SUSY breaking masses for $\mathcal{N}$ and $\overline{\mathcal{N}}$ whose square is assumed to be negative and $O\left(m_{3 / 2}^{2}\right)$, the scalar components of $\mathcal{N}$ and $\overline{\mathcal{N}}$ obtain the nonzero VEV such as $\langle\mathcal{N}\rangle=\langle\overline{\mathcal{N}}\rangle \sim \sqrt{m_{3 / 2} M}$. The bilinear $R$ parity violating term in $W_{3}$ is generated by this $\mathrm{VEV}$ from the first term in eq. (25) as $\epsilon=\langle\overline{\mathcal{N}}\rangle^{3} / M^{2} \cdot{ }^{10}$

The important problem is at what temperature the first term in eq. (25) leaves the thermal equilibrium. If the first term is assumed to leave the thermal equilibrium at $T_{X}$, the third term is also out of equilibrium but the second term still remains in the thermal equilibrium at $T_{X}$. This means that both $\mu_{\mathcal{N}}=\mu_{\overline{\mathcal{N}}}=0$ and $\mu_{L}+\mu_{\tilde{H}_{2}}=0$ can be satisfied at $T \gtrsim T_{X}$. This result is found to realize the same one which is given in eq. (16).

On the other hand, the effectively induced $W_{3}$ should be out of equilibrium at $T \gtrsim T_{W}$ since it violates the $L$ invariance. ${ }^{11}$ This gives the constraint on $\epsilon_{\alpha}$. It is convenient to redefine the chiral superfield $H_{1}$ as $H_{1}^{\prime} \equiv \frac{\epsilon_{\alpha}}{\mu} L_{\alpha}+H_{1}$ to estimate it. By this manipulation, $\epsilon_{\alpha} L_{\alpha} H_{2}$ disappears from the superpotential $W_{4}$ but there appear the new $R$-parity violating terms

$$
W_{\mathrm{RPV}}=-y_{E}^{\alpha \beta} \frac{\epsilon_{\gamma}}{\mu} L_{\alpha} \bar{E}_{\beta} L_{\gamma}-y_{D}^{\alpha \beta} \frac{\epsilon_{\gamma}}{\mu} Q_{\alpha} \bar{D}_{\beta} L_{\gamma}
$$

These interactions should not completely wash out the $B$ and $L$ asymmetries by the collaboration with the sphaleron interaction. The stringent constraint on these terms is derived by requiring that the $L$ violating scattering processes are out of thermal equilibrium at $T \gtrsim T_{W}$. By estimating these processes at $T_{W}$, we can find a condition such as $[13,27]$

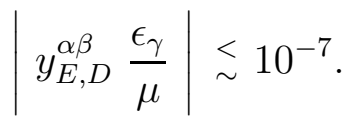

Since these Yukawa coupling constants $y_{E, D}$ are constrained by the masses of quarks and leptons, eq. (27) gives us a condition on $\left|\epsilon_{\alpha} / \mu\right|$. If we assume $\left\langle H_{1}^{\prime}\right\rangle=100 \mathrm{GeV}$, we find

\footnotetext{
${ }^{10}$ If $M$ is assumed to be an intermediate mass scale such as $M \gtrsim 5 \times 10^{9} \mathrm{GeV}$, we find that $\langle\overline{\mathcal{N}}\rangle \gtrsim 3 \times$ $10^{5} \mathrm{GeV}$ and $\epsilon \lesssim 7 \times 10^{-4} \mathrm{GeV}$. These values suggest that the neutrino masses based on the bilinear $R$-parity violation can be the dominant one for the neutrino masses in comparison with the usual seesaw contribution.

${ }^{11}$ This condition may be expressed that $H>\epsilon_{\alpha}^{2} / T$ is satisfied at $T \gtrsim T_{W}(\simeq 100 \mathrm{GeV})$.
} 
that $\left|\epsilon_{\alpha} / \mu\right| \lesssim 10^{-6} .{ }^{12}$ Since the constraint is very critical for this neutrino mass generation scheme in the present scenario, the more quantitative study seems to be necessary. In fact, in order to obtain the appropriate $B$ asymmetry in eq. (22), the substantial dilution $\kappa$ seems to be necessary for $N \geq 5$ as far as we do not require the extreme fine tuning of $\delta$.

The washout factor $\kappa$ can be quantitatively estimated by the analysis of the Boltzmann equation for the particle number density, which includes the effects of the $L$ violating two body scatterings. It can be written as $[1,27]$

$$
\frac{d N_{L}}{d x}=-\frac{\Gamma_{A}}{H(m)} \frac{x}{N_{L}^{\mathrm{EQ}}}\left(N_{L} N_{a}-N_{L}^{\mathrm{EQ}} N_{a}^{\mathrm{EQ}}\right)
$$

where $N_{f} \equiv n_{f} / s$ stands for the number density of $f$ per comoving volume and $N_{f}^{\mathrm{EQ}}$ represents the value at the thermal equilibrium. A dimensionless parameter $x=m / T$ is used for a certain mass scale $m$. $H(m)$ is the Hubble constant at $T=m$. Eq. (28) can be deformed into the equation for the $L$ asymmetry $Y_{L} \equiv N_{L}-N_{L^{c}}$. From this equation we can obtain the washout factor $\kappa$ as

$$
\kappa=\exp \left(-\int_{1}^{x_{0}} d x \frac{x \Gamma_{A}}{H(m)} \frac{Y_{a}^{\mathrm{EQ}}}{Y_{L}^{\mathrm{EQ}}}\right),
$$

where $x_{0}$ should be taken as a value at which the electroweak sphaleron interaction can be regarded to be out of equilibrium. If we assume that $m$ is the SUSY breaking scale $O(1) \mathrm{TeV}$ which seems to be a reasonable choice in the present scenario, $x_{0}$ should be fixed as 10 .

In the calculation of $\kappa$, the scattering process $L Q \rightarrow \lambda_{3} \bar{D}$ and its SUSY partner become the dominant contribution to the reaction rate $\Gamma_{A}$ in eq. (29). For these tree diagrams, it can be, respectively, represented as

$$
\Gamma_{A} \sim \alpha_{g} \alpha_{y_{E, D}}\left(\frac{\epsilon}{\mu}\right)^{2} \frac{T^{5}}{\left(T^{2}+m^{2}\right)^{2}}, \quad \Gamma_{A} \sim \alpha_{g} \alpha_{y_{E, D}}\left(\frac{\epsilon}{\mu}\right)^{2} T
$$

where we use $\alpha_{g}=g^{2} / 4 \pi$ and so on. For simplicity, we take the masses of all superpartners as $m$. Using these $\Gamma_{A}$ 's and practicing the numerical calculation of eq. (29), we find that $\kappa$ takes the values shown in Table 3 for various values of $|\epsilon / \mu|$. We also find that the value

\footnotetext{
${ }^{12}$ This constraint is much stronger than the ones obtained from the accelerator experiments [28]. The thermal corrections on the Higgsino masses is not expected to be so large as to make this bound change substantially.
} 


\begin{tabular}{cc|rc}
$|\epsilon / \mu|$ & $\kappa$ & $|\epsilon / \mu|$ & $\kappa$ \\
\hline $10^{-6}$ & 0.85 & $10^{-5.2}$ & $1.5 \times 10^{-3}$ \\
$10^{-5.5}$ & 0.2 & $10^{-5.1}$ & $3.5 \times 10^{-5}$ \\
$10^{-5.3}$ & $1.7 \times 10^{-2}$ & $10^{-5}$ & $8.6 \times 10^{-8}$ \\
\hline
\end{tabular}

Table 3 The washout effect $\kappa$ in the bilinear $R$-parity violating model.

of $\kappa$ becomes constant at $x_{0} \gtrsim 10$ for these values of $|\epsilon / \mu|$. It means that these $L$ violating

processes completely leave the thermal equilibrium at $T \sim T_{W}$. For $|\epsilon / \mu|>10^{-5}$, the value of $\kappa$ is less than $10^{-11}$ and we cannot obtain the sufficient $B$ asymmetry in any case. This estimation of $\kappa$ shows that the magnitude of the $R$-parity violating coupling is severely constrained by the washout effect. However, the neutrino mass generation scheme based on the bilinear $R$-parity violation seems to be compatible to the present scenario. This washout effect may make it possible to produce the appropriate $B$ asymmetry without any fine tuning of the value of $C P$ phases for a certain range of $|\epsilon / \mu|$.

\section{Summary}

We have proposed the baryogenesis scenario which is intimately related to the origin of the $\mu$-term and the small neutrino masses. If the $\mu$-term is assumed to be originated from a suitable VEV of the SM singlet field $S$ with the flat direction, the deviation of this condensate from the true vacuum value induces the coherent oscillation. If its nonrenormalizable interaction violates the global $\mathrm{U}(1)_{X}$ charge at the early stage of the universe and there is the non-adiabatic change of the $C P$ phase during the oscillation, the condensate can store the $X$ asymmetry due to the AD mechanism. Since its decay proceeds through the $\mathrm{U}(1)_{X}$ invariant interaction, this asymmetry is distributed into the thermal plasma. If a suitable interaction which violates both $\mathrm{U}(1)_{B-L}$ and $\mathrm{U}(1)_{X}$ is in the thermal equilibrium at the temperature $T_{X}$ where the decay of condensate has been almost completed, this $X$ asymmetry can be converted into the $L$ and $B$ asymmetry.

The required condition for the success of this scenario is that the temperature $T_{X}$ should be higher than $T_{s s}$ at which the soft SUSY breaking terms come in the thermal equilibrium. Moreover, the interaction violating both $\mathrm{U}(1)_{B-L}$ and $\mathrm{U}(1)_{X}$ should leave 
the thermal equilibrium at the temperature $T$ such as $T_{s s} \lesssim T \lesssim T_{X}$. If these conditions are satisfied, the conversion of the $X$ asymmetry into the $B$ asymmetry can be substantially proceeded. The produced abundance of the $B$ asymmetry can be related to the dimension of the $\mathrm{U}(1)_{X}$ violating interaction. We have also studied the compatibility with two types of $L$ violating interactions which are intimately related to the small neutrino mass generation.

We have left some problems as those beyond the scope of this paper. One of them is to present the concrete models which cause the final stage symmetry breaking for $S$ at a required scale. In [25] this kind of study has been done extensively for the similar models in the different context, and they found that the symmetry breaking scale required here could be successfully realized. We also have not sufficiently discussed the relation between this scenario and the inflation of the universe as the next step problem. It is necessary to embed this scenario into a suitable inflation model. We may construct such a kind of inflation model in the direction of [17, 29], in which the similar models are discussed. These subjects will be discussed in other places.

This work is supported in part by a Grant-in-Aid for Scientific Research (C) from Japan Society for Promotion of Science (No. 14540251) and also by a Grant-in-Aid for Scientific Research on Priority Areas (A) from The Ministry of Education, Science, Sports and Culture (No. 14039205). 


\section{Appendix A}

The nonrenormalizable term in the superpotential $W_{1}$ can be controlled by introducing a suitable symmetry. One way is to impose a discrete symmetry $Z_{N}$ and assign its unit charge to $S$. Then the second term in $W_{1}$ can be obtained as the lowest order term. We can also obtain other terms in $W_{\mathrm{MSSM}}+W_{1}$ by assigning the discrete charge to the MSSM contents suitably. This construction is assumed in the text.

It may be extended by introducing one more singlet chiral superfield $\bar{S}$ and considering the discrete symmetry $Z_{N^{\prime}}\left(N^{\prime}>N\right)$. We assign them the discrete integer charge as $q(>0)$ for $S$ and $-p(<0)$ for $\bar{S}$. If the least common multiple for $p$ and $q$ is assumed to be $p q$ and $p+q=N$, the lowest order $Z_{N^{\prime}}$ invariant term is $\frac{d}{M_{\mathrm{pl}}^{N-3}} S^{p} \bar{S}^{q}$. Considering a $D$-flat direction: $\langle S\rangle=u e^{i \theta_{S}},\langle\bar{S}\rangle=u e^{i \theta_{\bar{S}}}$ and putting $\theta=p \theta_{S}+q \theta_{\bar{S}}$, then the feature of this direction is similar to that discussed in the text. However, in this case we needs to introduce a coupling of $\bar{S}$ to some chiral superfields $f_{\alpha}$ which makes the decay of the $\bar{S}$ condensate possible. As far as the MSSM fields have no interaction with $f_{\alpha}$, the same results obtained in the text will be realized. The decay products $f_{\alpha}$ might explain the cold dark matter abundance if they have no other interaction which breaks the $X$ symmetry.

Another way to present $W_{1}$ is to assume the existence of an additional $\mathrm{U}(1)$ gauge symmetry to the MSSM gauge structure. We assign its integer charge with the opposite sign such as $q(>0)$ and $-p(<0)$ to $S$ and $\bar{S}$ where $p$ and $q$ is assumed to satisfy the condition that their least common multiple is $p q$ and $p+q=N$. Then the same order term $\frac{d}{M_{\mathrm{pl}}^{N-3}} S^{p} \bar{S}^{q}$ to the second one in $W_{1}$ can be obtained as the lowest order term constructed from $S$ and $\bar{S}$. The introduction of such a symmetry is also favored from a view point to escape the tadpole problem and the domain wall problem, which are expected to appear often in the models extended only by the singlet chiral superfield [24]. Moreover, since the extra $\mathrm{U}(1)$ gauge interaction imposes the $D$-flatness $q|\langle S\rangle|=p|\langle\bar{S}\rangle|$, both condensate $S$ and $\bar{S}$ can decay through the coupling $\lambda S H_{1} H_{2}$. In this case, however, the introduction of the new fields is required from the gauge anomaly cancellation. Since these fields contribute to the thermal equilibrium conditions for the $\mathrm{SU}(2)$ and $\mathrm{SU}(3)$ sphaleron interactions etc., the discussion presented in section 3 cannot be directly applied. Although it may be possible to introduce the new fields without changing the results obtained in 
the text qualitatively, the model will be rather complicated.

\section{Appendix B}

In this appendix we briefly explain the small neutrino mass generation in the $R$-parity violating models. In the MSSM extended by $W_{3}$, the first term in $W_{1}$ or the $\mu$-term collaborates with $W_{3}$ to induce a mixing mass matrix between neutrinos and neutralinos in such a way as

$$
\begin{aligned}
& \mathcal{M}=\left(\begin{array}{cc}
0 & M_{m} \\
M_{m} & M_{\mathcal{N}}
\end{array}\right), \quad M_{m}=\left(\begin{array}{cccc}
\sqrt{2} g_{2}\left\langle\tilde{\nu}_{e}\right\rangle & \sqrt{2} g_{1}\left\langle\tilde{\nu}_{e}\right\rangle & 0 & \epsilon_{e} \\
\sqrt{2} g_{2}\left\langle\tilde{\nu}_{\mu}\right\rangle & \sqrt{2} g_{1}\left\langle\tilde{\nu}_{\mu}\right\rangle & 0 & \epsilon_{\mu} \\
\sqrt{2} g_{2}\left\langle\tilde{\nu}_{\tau}\right\rangle & \sqrt{2} g_{1}\left\langle\tilde{\nu}_{\tau}\right\rangle & 0 & \epsilon_{\tau}
\end{array}\right), \\
& M_{\mathcal{N}}=\left(\begin{array}{cccc}
M_{2} & 0 & \frac{1}{\sqrt{2}} g_{2} v_{1} & -\frac{1}{\sqrt{2}} g_{2} v_{2} \\
0 & M_{1} & -\frac{1}{\sqrt{2}} g_{1} v_{1} & \frac{1}{\sqrt{2}} g_{1} v_{2} \\
\frac{1}{\sqrt{2}} g_{2} v_{1} & -\frac{1}{\sqrt{2}} g_{1} v_{1} & 0 & \mu \\
-\frac{1}{\sqrt{2}} g_{2} v_{2} & \frac{1}{\sqrt{2}} g_{1} v_{2} & \mu & 0
\end{array}\right),
\end{aligned}
$$

where we write $\mathcal{M}$ by using the basis $\left(L_{\alpha},-i \lambda_{2}^{3},-i \lambda_{1}, \tilde{H}_{1}, \tilde{H}_{2}\right) . M_{\mathcal{N}}$ corresponds to the usual neutralino mass matrix. Nonzero sneutrino VEVs $\left\langle\tilde{\nu}_{\alpha}\right\rangle$ are expected to be derived by minimizing the scalar potential which contains the soft SUSY breaking terms $B_{\epsilon_{\alpha}} \epsilon_{\alpha} L_{\alpha} H_{2}$ corresponding to $W_{3}$. It can be estimated as $\left\langle\tilde{\nu}_{\alpha}\right\rangle \sim O\left(\epsilon_{\alpha}\right)$. If we assume that both the absolute values of $\epsilon_{\alpha}$ and $\left\langle\tilde{\nu}_{\alpha}\right\rangle$ are much smaller than $\mu$ and $M_{1,2}$ which are considered to be the order of weak scale $M_{W}$, the small neutrino masses are generated by the weak scale seesaw mechanism $[18,19]$.

It is easily checked that $\mathcal{M}$ has two zero and five nonzero eigenvalues. The four nonzero eigenvalues correspond to those of the neutralinos and they are $O\left(M_{W}\right)$. On the other hand, as discussed in [19], the tree-level mass eigenvalues are characterized by the quantities $\Lambda_{\alpha} \equiv \epsilon_{\alpha}\left\langle H_{1}\right\rangle+\mu\left\langle\tilde{\nu}_{\alpha}\right\rangle$ in the effective neutrino mass matrix. Using this $\Lambda_{\alpha}$, the smallest one of the nonzero eigenvalues is written as $\left(M_{1} g_{1}^{2}+M_{2} g_{2}^{2}\right)|\vec{\Lambda}|^{2} / 4 \operatorname{det}\left(M_{\mathcal{N}}\right)$. The remaining massless states are known to become massive by taking account of the radiative corrections. If we impose the constraints on the mass and mixing required for the explanation of the neutrino oscillation data $[4,5]$, it is shown that $\epsilon$ should satisfy the condition such as $|\epsilon / \mu| \sim O\left(10^{-4 \sim-3}\right)$ by using the numerical analysis [19].

The model with the bilinear $R$-parity violating term can be extended by introducing the generation dependent extra $\mathrm{U}(1)$ gauge symmetry at the $\mathrm{TeV}$ region [20]. In this 
case, the neutrino mass degeneracy can be resolved at the tree-level and then the mass and mixing of neutrinos can be directly related to $\epsilon$. The largest mass eigenvalue can be written as $O\left(\left|\epsilon^{2} / \mu\right|\right)$, where the gaugino masses are assumed to be $O(|\mu|)$. By imposing the condition for the $\nu_{\tau}$ mass required from the explanation of the atmospheric neutrino anomaly, we can obtain a condition $|\epsilon / \mu| \sim O\left(10^{-6}\right)$ for a reasonable value of $\mu$. The value of $|\epsilon|$ can be smaller than the one in the previous example by two or three order of magnitude. 


\section{References}

[1] For reviews, see for example, E. Kolb, M. S. Turner, The Early Universe, AddisonWesley, New York,1990; A. D. Dolgov, Phys. Rep. 222c (1992) 309; A, Riotto and M. Trodden, Ann. Rev. Nucl. Part. Sci. 49 (1999) 35.

[2] V. A. Kuzmin, V. A. Rubakov and M. E. Shaposhnikov, Phys. Lett. B155 (1985) 36.

[3] For a recent review, see for example, W. Buchmüller, hep-ph/0204288

[4] Y. Suzuki, Super-Kamiokande Collaboration, Nucl. Phys. B (Proc. Suppl.)91 (2001) 29.

The SNO Collaboration, Phys. Rev. Lett. 87 (2001) 071301; Phys. Rev. Lett. 89 (2002) 011302; Phys. Rev. Lett. 89 (2002) 011301.

The KamLAND Collaboration, Phys. Rev. Lett. 90 (2003) 021802; 92 (2004) 071301; hep-ex/0406035.

[5] Super-Kamiokande Collaboration, Y. Fukuda et at., Phys. Rev. Lett. 81 (1998) 1562; Phys. Lett. B436 (1998) 33; Phys. Lett. B433 (1998) 9.

The K2K Collaboration, Phys. Rev. Lett. 90 (2003) 041801; Phys. Rev. Lett. 93 (2004) 051801.

[6] M. Fukugita and T. Yanagida, Phys. Lett. B174 (1986) 45.

[7] I. Affleck and M. Dine, Nucl. Phys. B249 (1985) 361.

[8] H. Murayama and T. Yanagida, Phys. Lett. B322 (1994) 349; M. Dine, L. Randall and S. Thomas, Nucl. Phys. B458 (1996) 291.

[9] H. Murayama, H. Suzuki, T. Yanagida and J. Yokoyama, Phys. Rev. Lett. 70 (1993) 1912.

[10] D. Suematsu and Y. Yamagishi, Mod. Phys. Lett. A38 (1995) 2923.

[11] S. M. Barr, R. S. Chivukula and E. Farhi, Phys. Lett. B241 (1990) 387; D. B. Kaplan, Phys. Rev. Lett. 68 (1992) 741. 
[12] L. E. Ibáñez and F. Quevedo, Phys. Lett. B283 (1992) 261.

[13] H. Dreiner and G. G. Ross, Nucl. Phys. B410 (1993) 188.

[14] J. E. Kim and H. P. Nilles, Phys. Lett. 138B (1984) 150.

[15] For a recent review, see for example, N. Polonsky, hep-ph/9911329 and references therein.

[16] T. Gherghetta and G. L. Kane, Phys. Lett. B354 (1995) 300; M. Bastero-Gil and S. F. King, Phys. Lett. 423 (1998) 27; G. L. Kane and S. F. King, New J. Phys. 3 (2001) 21.

[17] G.R. Dvali, G. Lazarides and Q. Shafi, Phys. Lett. B424 (1998) 259; G. Lazarides and N.D. Vlachos, Phys. Lett. B459 (1999) 482.

[18] F. de Compos, M. A. Garcá-Jareño, A. S. Joshipura, J. Rosiek and J. W. F. Valle, Nucl. Phys. B451 (1995) 3; T. Banks, Y. Grossman, E. Nardi and Y. Nir, Phys. Rev. D52 (1995) 5319; A. S. Joshipura and M. Nowakowski, Phys. Rev. D51 (1995) 2421; M. A. Díaz, J. C. Romão and J. W. F. Valle, Nucl. Phys. B524 (1998) 23; J. W. F. Valle, in Pro. 6th International Symposium on Particles, Strings and Cosmology (PASCOS 98), Boston 1998, p502 and references therein.

[19] M. Hirsch, M. A. Díaz, W. Porod, J. C. Romão and J. W. F. Valle, Phys. Rev. D62 (2000) 113008.

[20] D. Suematsu, Phys. Lett. B506 (2001) 131; Phys. Rev. D64 (2001) 073013. J. Kubo and D. Suematsu, Phys. Rev. D64 (2001) 115014.

[21] M. Dine, L. Randall and S. Thomas, Phys. Rev. Lett. 75 (1995) 398. M. Dine, L. Randall and S. Thomas, Nucl. Phys. B458 (1996) 291.

[22] R. J. Scherrer and M. S. Turner, Phys. Rev. D31 (1985) 681.

R. Allahverdi, B. A. Campbell and J. Ellis, Nucl. Phys. B579 (2000) 355.

A. Anisimov and M. Dine, Nucl. Phys. B619 (2001) 729.

[23] J. Ellis, A. Linde and D. Nanopoulos, Phys. Lett. B118 (1982) 59. 
[24] D. Suematsu and Y. Yamagishi, Int. J. Mod. Phys. A10 (1995) 4521; M. Cvetič and P. Langacker, Phys. Rev. D54 (1996) 3570; D. Suematsu and G. Zoupanos, J. High Energy Phys. 06 (2001) 038.

[25] G. Cleaver, M. Cvetič, J. R. Espinosa, L. Everett and P. Langacker, Phys. Rev. D57 (1998) 2701.

[26] S. Yu. Khlebnikov and M. E. Shapshnikov, Nucl. Phys. B308 (1988) 885; J. A. Harvey and M. S. Turner, Phys. Rev. D42 (1990) 3344;

[27] W. Buchmüller and M. Plümacher, Int. J. Mod. Phys. A15 (2000) 5047, and references therein.

[28] R. Barbier, et al., hep-ph/9810232.

[29] D. Suematsu, JHEP 10 (2002) 014. 